\title{
LAS ACTITUDES HACIA LAS NECESIDADES EDUCATIVAS ESPECIALES EN FUNCIÓN DEL \\ GÉNERO: IMPLEMENTACIÓN Y EVALUACIÓN DE UN PROGRAMA DE APS \\ EN EL ÁMBITO UNIVERSITARIO
}

\author{
Marta Liesa Orús \\ martali@unizar.es \\ Universidad de Zaragoza \\ Pilar Arranz Martínez \\ parranz@unizar.es \\ Universidad de Zaragoza
}

\author{
Sandra Vázquez Toledo \\ svaztol@unizar.es \\ Universidad de Zaragoza \\ Pilar Otal Piedrafita \\ pilotal@unizar.es \\ Universidad de Zaragoza
}

Recibido: 28-02-2014

Aceptado: 09-04-2014

\begin{abstract}
Resumen
En esta investigación analizamos los resultados de la implementación de un programa educativo. Este programa pretende completar la formación en atención a la diversidad de los alumnos y alumnas del Grado de Maestro de Educación Primaria apoyándose en la metodología de aprendizaje servicio. Los resultados muestran que los alumnos de Magisterio varones presentan actitudes menos positivas hacia la inclusión de las personas con necesidades educativas especiales, basadas en la teoría y en el modelo del déficit. Estas actitudes se corrigen cuando se ha implementado el programa a través de las estrategias de formación y contacto con personas con discapacidad. Planteamos una investigación cuasi-experimental pretest-postest con grupo de control no equivalente, la muestra son los alumnos y alumnas de segundo de Magisterio del curso 2012-2013.
\end{abstract}

Palabras Clave: Necesidades educativas especiales, universidad, género, aprendizaje servicio.

\begin{abstract}
In this research we analyze the results of the implementation of an educational program. This program aims to complete, relying on the service learning methodology, the training on attention to the diversity of students of the Teacher Degree. The results show that male students have less positive attitudes toward inclusion of persons with special educational needs, based on the theory and the deficit model. These attitudes are corrected when the program is implemented through training strategies and with contact with persons with disabilities. We propose a quasi-experimental research with pretest-posttest and non-equivalent control group, in which the sample were the students of second school year of the Teacher Degree 2012-2013.
\end{abstract} Keywords: Special educational needs, university, gender, service learning. 


\section{Introducción}

Esta investigación se plantea analizar los resultados de la implementación de un proyecto que hemos desarrollado en la Facultad de Ciencias Humanas y de la Educación de Huesca, en la asignatura de Atención a la Diversidad de segundo curso del Grado de Magisterio en Educación Primaria; basado en la metodología de aprendizaje-servicio. El principal objetivo es completar la formación de los estudiantes en este área y mejorar las diferencias de actitudes hacia las necesidades educativas especiales existentes según el sexo de los estudiantes que habían sido detectadas en el pretest.

Para ello los alumnos han recibido una formación teórico-práctica de 80 horas, de las cuales sesenta son formación teórica y se trabajan en la propia asignatura y veinte horas de prácticas que permiten que nuestros estudiantes compartan actividades con niños y jóvenes con necesidades educativas especiales. Estas prácticas se realizan en diferentes escenarios de inclusión vinculados a las asociaciones de la Coordinadora de Asociaciones de Discapacidad de Huesca (en adelante CADIS) en la que se integran, entro otras: Asociación Down, ASPACE, ATADES, Asociación de Hipoacúsicos. Dicha formación práctica se ha concretado en sesiones de dos horas semanales.

Nos planteamos este proyecto pensando que favorecería un cambio de actitud positiva por parte de los futuros maestros y así mismo disminuirían las diferencias actitudinales que se encontraban en la variable género, hecho que ha sido constatado con los resultados. Apostamos por esta formación pues estamos convencidos de que nos conducirá a escuelas más inclusivas y en general, a una sociedad mucho más integradora, con mayor capacidad para ofrecer a las personas con necesidades educativas especiales entornos que favorezcan su autodeterminación, su vida independiente y en definitiva una mayor calidad de vida.

\section{Revisión de Investigaciones}

La investigación más prolífica sobre los factores que influyen en la actitud de los maestros hacia la integración de las personas con necesidades educativas especiales aparece a partir de los años setenta en el ámbito anglosajón, gracias a la influencia del Informe Warnock 
(1978) y los constructos de normalización e integración. En España, es a partir de los años 80, cuando se empieza a trabajar en el ámbito de la evaluación y el cambio de actitudes.

El interés por el tema radica en que las actitudes de los profesores tienen un efecto importante no sólo en el desarrollo del autoconcepto del propio sujeto con necesidades educativas especiales, sino también en su proceso de socialización, de integración y actualmente de inclusión. "Estas actitudes cuando son negativas, constituyen una de las principales barreras en la integración social de este colectivo" (Flórez, León Aguado, y Alcedo, 2009: 85).

En este sentido podemos citar las aportaciones de Pelechano (1990) y Pelechano, Peñate y De Miguel (1991), Pelechano, García y Hernández (1994) en las que se muestra la eficacia de la evaluación de las actitudes ante la integración de discapacitados visuales como paso previo a su integración. Similares aportaciones encontramos en Dengra, Durán y Verdugo (1991) referidas a la integración de escolares con necesidades educativas especiales, en Gómez e Infante (2004) que estudian las actitudes de estudiantes universitarios hacia personas con discapacidad y minorías étnicas, así como en Arias (1994), Sáenz (1990), Verdugo, Arias y Jenaro (1994) y Surriá (2011) alusivas a la integración de necesidades educativas especiales en general.

Algunos estudios relacionan las actitudes hacia las necesidades educativas especiales con la variable género. Entre estos estudios cabe destacar los realizados por Downs y Wiliams, 1994; Folsom-Meek y Rizzo, 2002; Infante y Gómez, 2004; Novo y Muñoz, 2012, que han encontrado diferencias significativas en cuanto a la actitud hacia las necesidades educativas especiales entre hombres y mujeres, siendo éstas últimas más sensibles.

A pesar de su importancia, no es frecuente que las actitudes sean tenidas en cuenta en los currículos y proyectos docentes destinados a estudiantes que una vez egresados serán profesionales de la docencia. Así, Verdugo, Jenaro y Arias (1995: 152) señalan que: “a pesar de la importancia primordial de las actitudes hacia las personas con necesidades educativas especiales para lograr una integración social real, todavía son pocos los centros e instituciones que incluyen, como parte importante de su quehacer profesional, actividades, objetivos y contenidos dirigidos a evaluar y mejorar las actitudes".

Sin embargo, en la actualidad existen numerosas técnicas e instrumentos para la valoración y cambio de las actitudes. Entre estos trabajos destacan los de Verdugo, Jenaro y Arias (1995) que desarrollaron una "Escala de Actitudes hacia las Personas con Discapacidad" 
(EAPD). Ésta incluye una forma G (general) con la que pueden valorarse las actitudes frente a personas afectadas de cualquier necesidad educativa especial, y formas específicas de esta misma escala para la valoración de actitudes ante personas con discapacidad física, sensorial y mental, destinadas tanto a adolescentes como a adultos.

Flórez, León y Alcedo (2009), hacen una revisión de varios programas diseñados para el cambio de actitudes hacia personas con necesidades educativas especiales desde los años 70 a la actualidad, y hemos podido observar como la mayor parte de los programas utilizan como estrategia más frecuente para desarrollar actitudes positivas hacia las personas con discapacidad, el contacto directo con las personas con necesidades educativas especiales, la formación o la combinación de las dos técnicas.

El contacto con personas con necesidades educativas especiales, es una técnica que se basa en el contacto programado que puede ser dentro de la escuela o fuera de ella, en actividades formativas o de ocio, como salidas, excursiones, etc. La mayoría de investigaciones muestran que el contacto es una técnica potente para provocar el cambio hacia actitudes más positivas. Las puntuaciones en el grupo experimental mejoran respecto al grupo control y adquieren significación estadística en los análisis inter e intragrupo. Se recomienda que dicho contacto sea muy estructurado, es decir que el tipo de actividad, el momento y el lugar de contacto estén previamente planificados. Entre las investigaciones que ratifican esta idea se encuentran las siguientes:

- Evans en 1976 hizo un estudio con una muestra de sesenta estudiantes universitarios. Analizaba dos grupos de estudiantes, uno que realizaba contactos estructurados con personas con discapacidad visual y otros que también realizaban contactos pero estos no eran estructurados. Los resultados fueron que hubo cambios positivos en el grupo de contactos estructurados y no en el otro.

- Craig en 1988, también analizó un estudio con una muestra de 34 estudiantes universitarios, a los que se les facilitó contacto con chicos con paraplejia. Se les pasó luego una escala tipo Likert en dos momentos (pretest-postest). En los resultados se observó un cambio positivo de actitudes en el grupo experimental.

- Avramidis y Norwichi (2004), hacen una revisión exhaustiva sobre la actitud de los profesores de educación general no especialistas ante la integración y la inclusión y analizan los diferentes factores que podrían influir sobre la formación de estas actitudes, 
siendo uno de estos factores el contacto directo con alumnos con necesidades educativas especiales.

- Sales, A.; Moliner, O. y Sanchiz, M ${ }^{\mathrm{a}}$.L., 2001 refieren un estudio realizado con estudiantes de magisterio, en el que se analizan las actitudes positivas hacia la diversidad y la inclusión del alumnado con necesidades educativas especiales. Es únicamente un estudio descriptivo que demuestra que tras pasar un cuestionario a los estudiantes se confirman algunas actitudes negativas hacia la integración, la inclusión y la diversidad basadas en la teoría y el modelo del déficit. Los autores proponen cambios metodológicos en la formación de los maestros basados en la información extra, en las prácticas y en experiencias vinculadas a la educación especial.

- Otro estudio reciente se ha realizado en la Universidad de Sevilla con una muestra de 498 estudiantes de diferentes titulaciones todas ellas relacionadas con la educación: Magisterio, Psicopedagogía, Educación Especial, Psicología, etc. Se les pasó la misma escala utilizada en la investigación descrita en este artículo, para analizar la actitud hacia las personas con discapacidad. El resultado más importante al que han llegado es que aquellos estudiantes que tienen contacto con personas con discapacidad en su vida tienen una actitud más positiva hacia ellas (Moreno, Rodriguez, Saldaña y Aguilera, 2006).

Además del contacto directo, otra técnica que ha tenido efectos positivos en el cambio de actitudes hacia la inclusión social de las personas con necesidades educativas especiales ha sido la formación variada acerca de cuestiones relacionadas con el ámbito de la discapacidad. Esta información se puede ofrecer al grupo experimental de manera directa (a través de las mismas personas con discapacidad, o expertos en el tema), de manera indirecta (películas, libros, documentales, etc.) o de forma mixta (conjugando las dos anteriores). Siempre es conveniente que esta técnica vaya acompañada de la discusión guiada a lo largo de todo el programa por el experto que aporta la información. Hay numerosos estudios de cómo influye esta técnica de manera muy positiva en la actitud de los estudiantes de primaria, pero pocos en relación a cómo influye en los estudiantes universitarios. Lazar, Orpet y Demos (1976) que utilizaron esta técnica con un grupo de 20 estudiantes de Máster Universitario, dándoles información a través de conferencias y lecturas. Aquí sí que se observó una mejora de actitudes 
hacia las personas con necesidades educativas especiales al comparar las respuestas en una escala Likert que se cumplimentó antes y después del tratamiento formativo.

La técnica que parece que en las investigaciones realizadas ha obtenido mejores resultados en la modificación de actitudes positivas hacia la inclusión de las personas con necesidades educativas especiales es la utilizada en esta investigación, que se podría denominar "la información más el contacto", es la combinación de las dos técnicas anteriormente expuestas y parece ser la técnica más eficaz. Skrtic, Clark y White realizado en 1982, un estudio con una muestra de 109 alumnos de magisterio divididos en dos grupos, uno recibió información sobre personas con discapacidad visual, y el otro grupo recibió información más contacto directo con personas con discapacidad visual y los resultados muestran que mejoraron las actitudes hacia las personas con necesidades educativas especiales en ambos grupos y que hubo más ganancia en el grupo en el que se utilizó la técnica de información más contacto.

Otra cuestión interesante, e incluso novedosa de nuestra investigación es que para poner en marcha la estrategia de la formación más el contacto de nuestros estudiantes con personas con discapacidad nuestro proyecto se apoya en la metodología del Aprendizaje Servicio (en adelante ApS). El Aprendizaje-Servicio es un metodología pedagógica basada en la experiencia (Stanton, 1990), en la cual los estudiantes, docentes y miembros de una institución comunitaria o pública trabajan juntos para satisfacer las necesidades de esa comunidad; integrando y aplicando conocimientos académicos, alcanzando de esta forma, los objetivos instruccionales del curso. Para esto se usa la acción, la reflexión crítica y la investigación; y se forma a los estudiantes para convertirse en miembros contribuyentes a una sociedad más justa y democrática (Stephenson, Wechsler y Welch, 2002).

Coincidimos con Rial (2010) al entender el aprendizaje-servicio como una propuesta pedagógica que implica la realización de una acción solidaria protagonizada por los/las estudiantes, destinada a atender necesidades reales de una comunidad y planificada en forma integrada con los contenidos curriculares de aprendizaje.

Siguiendo esta metodología, para poner en marcha nuestro proyecto nos reunimos con las asociaciones con discapacidad de la provincia. Expusieron sus necesidades actuales y analizamos conjuntamente el servicio que podían ofrecer nuestros estudiantes para ayudar a cubrir dichas necesidades mediante actividades prácticas que a su vez les ayudasen a adquirir las competencias profesionales del título. A partir de esa reflexión se diseñaron veinte horas de prácticas en las asociaciones. Estas prácticas cumplían un doble objetivo: ofrecer ApS de 
servicio a la comunidad y a la vez contribuir a la adquisición de las competencias curriculares de la asignatura Atención a la Diversidad y de otras materias del Grado de Maestro.

Por lo tanto, el ApS consiste en una propuesta educativa que combina procesos de aprendizaje y de servicio a la comunidad en un solo proyecto bien articulado, donde los participantes aprenden al trabajar en necesidades reales del entorno con la finalidad de mejorarlo (Puig y Palos, 2006). Además, el Aprendizaje Servicio sin dejar de ser un programa, es también una filosofía. Es decir, una manera de entender el crecimiento humano, una manera de explicar la creación de vínculos sociales y un camino para construir comunidades humanas más justas y con una mejor convivencia (Puig et. al, 2007).

Como vemos, los proyectos de aprendizaje-servicio permiten desarrollar los contenidos curriculares, es decir, aprendizajes académicos, pero además de una manera más clara y sencilla de lo que ocurre con otras metodologías, facilita la práctica de la planificación, el esfuerzo, la responsabilidad, el compromiso solidario... un sinfín de valores que de otra forma resulta complicado poder abordar desde una asignatura; a su vez, activa el ejercicio de todas las competencias del currículum, con énfasis en la iniciativa y autonomía personal y la competencia social y ciudadana. Valores y competencias todos ellos muy relacionados con el cambio de actitudes hacia una sociedad más solidaria e inclusiva, como en el fondo pretende nuestra investigación. Como puede apreciarse el aprendizaje aporta calidad al servicio y el servicio aporta significado y aplicación al aprendizaje (Tapia, 2000, 2008). El ApS tal y como indica Furco (2004) está orientado explícita y planificadamente a ofrecer un servicio solidario eficaz y a mejorar el aprendizaje de los estudiantes. Además, esta metodología logra aumentar significativamente los niveles de retención y aplicación de los contenidos de los cursos que la utilizan versus los que no lo hacen (Astin et al., 2000).

Con independencia del contenido específico que aborde cada una de las experiencias concretas, las actividades de aprendizaje-servicio comparten determinadas condiciones pedagógicas, entre las que destacan: el aprendizaje a partir de la experiencia, el aprendizaje cooperativo, la reflexión sobre la acción y la guía que ofrecen los adultos (Puig Rovira et al., 2011).

Estas condiciones son las que tuvimos en cuenta a la hora de proponer este proyecto a nuestros estudiantes. Por eso simultáneamente y también después de que los estudiantes aprendieran en la práctica real en las asociaciones en contacto directo con personas con discapacidad, compartíamos dichas experiencias en las clases teóricas de la asignatura, y 
reflexionábamos sobre las prácticas realizadas y a través del trabajo cooperativo encontrábamos mejoras en dicha práctica y la enriquecíamos, y además el profesorado actuaba como guía de los estudiantes de magisterio, y se les ayudaba a solucionar las dudas que tenían los estudiantes en el contacto con personas con necesidades educativas especiales, para que pudieran ofrecer la máxima ayuda a las personas con necesidades educativas especiales. Y a la vez se les ponía en condiciones de alcanzar el mayor número de competencias curriculares de la titulación.

Esta propuesta entiende el aprendizaje como un proceso que se basa en la exploración, la acción y la reflexión, como método para demostrar la aplicabilidad de lo aprendido; y considera que la educación en valores debe partir de situaciones problemáticas para enfrentarse a ellas desde la experiencia directa (Ferrán y Guinot, 2012)

Se trata, en definitiva, de una oportunidad excelente para tejer redes de colaboración y corresponsabilidad entre la universidad y las organizaciones sociales ubicadas en un territorio determinado con el fin de establecer procesos de aprendizaje significativo y de cohesión social (Ferrán y Guinot 2012).

\section{Diseño y Planificación del Proyecto}

Nuestra experiencia tiene dos objetivos claros: por un lado formar maestros y maestras competentes para trabajar en escuelas inclusivas, y por otro mejorar la calidad de vida de las personas con necesidades educativas especiales ayudando a su inclusión educativa y social. Para llevar a cabo estos dos objetivos se han buscado escenarios del entorno próximo dónde los estudiantes estén en contacto e interactúen con personas con necesidades educativas especiales a través del aprendizaje servicio.

El aprendizaje servicio incluye propuestas pedagógicas que intentan ayudar a disminuir las necesidades sociales existentes mejorando la calidad de vida de la comunidad. Es decir, en el caso que nos ocupa, supone el desarrollo de propuestas que favorezcan la inclusión social y la participación ciudadana de personas con necesidades educativas especiales, que es uno de los objetivos básicos para conseguir desarrollar en ellos una calidad de vida razonable. Como ciudadanos tienen el derecho de participar en su comunidad. Para ello la comunidad tiene que facilitar entornos sociales accesibles. 
Pero la situación actual está todavía muy lejana de esa concepción. Es preciso abrir nuevas vías de actuación que incidan en los entornos y que permitan aminorar la distancia entre la actual situación y la que desearíamos para ellos, centrada en el respeto a la diferencia, en la aceptación, la adaptabilidad de los entornos y la accesibilidad. Es preciso, por tanto, indagar y definir nuevos escenarios que faciliten la inclusión social de las personas con discapacidad para mejorar la calidad de vida de estas personas.

Una vez detectada la necesidad social, y ligada a la obligación de la Universidad de tender al bien común y favorecer la inclusión social de todos los ciudadanos, se vinculó dicho objetivo a contenidos conceptuales, procedimentales y actitudinales y a competencias que deberían adquirir los estudiantes en las diferentes asignaturas de la titulación de Maestro, en concreto en la investigación que estamos exponiendo en la asignatura de Atención a la Diversidad.

\subsection{Estrategias para modificar actitudes}

1. Contacto con personas con necesidades educativas especiales: A través de encuentros programados y estructurados con personas con algún tipo de necesidades educativas especiales, a través de excursiones, salidas, juegos, vivienda, piscina, ocio, etc. Diferentes estudios muestran que el contacto directo es una técnica válida y útil.

2. Información. A través de esta técnica se aporta información variada acerca de cuestiones relacionadas con el ámbito de la atención a la diversidad. Esta información se la proporcionamos a través de las sesenta horas de la asignatura de Atención a la Diversidad, utilizando diferentes recursos, explicación del profesor, o la participación a través de charlas de profesionales que están trabajando con personas con discapacidad en las asociaciones, o bien usando materiales variados: como vídeos, libros, artículos, películas, documentales, etc. relacionados con la discapacidad. También nosotros "como expertos" favorecemos posteriormente la discusión del grupo, la vivenciación de situaciones, o bien la resolución de una serie de tareas a través del trabajo en grupos cooperativos.

3. Trabajo cooperativo. Entre estudiantes de magisterio y personas con necesidades educativas especiales. Estas estrategias se ponen en marcha en las diferentes asociaciones dónde nuestros estudiantes acuden para mejorar la formación inicial y las actitudes de los estudiantes de magisterio hacia la diversidad y para mejorar la calidad de vida de las personas con necesidades 
educativas especiales. En este estudio sólo pretendemos analizar las variables relacionadas con los estudiantes de magisterio.

Los estudiantes en las distintas asociaciones participan en diferentes programas con personas con necesidades educativas especiales como puede ser: ayudar en la elaboración de material didáctico y creación literaria, colaborar en el programa de radio, en el proyecto vida independiente (piso de estudiantes en el que conviven estudiantes universitarios y jóvenes con discapacidad), en atención temprana, en actividades de ocio, deportivas (como la piscina), en excursiones, en implementación de programas de desarrollo cognitivo, programas de desarrollo de habilidades prácticas de la vida diaria (en cocina, educación vial, manejo del dinero), etc.

Los estudiantes pasan veinte horas colaborando en la asociación que han elegido en un determinado programa o en varios de los que nos oferta la asociación y cooperando con los trabajadores de la asociación y con las personas con necesidades educativas especiales en las necesidades que tienen en ese momento. A la vez el estudiante en las sesenta hora de formación teórica en las clases de la Universidad está recibiendo la formación inicial que necesita para afrontar este precioso reto, y como la formación teórica y la experiencia práctica se dan a la vez, los estudiantes pueden traer a clase las dudas que les surgen colaborando en las asociaciones con personas con necesidades educativas especiales y el profesor las resuelve, o genera debates en grupo, o se resuelven a través de técnicas de trabajo cooperativo, etc.

\subsection{Planteamiento del problema}

Tras una breve introducción teórica, el problema queda definido de la siguiente forma: “ $L a$ formación en atención a la diversidad de los estudiantes de magisterio es muy escasa y esto puede influir en su actitud hacia la inclusión educativa y social de las personas con necesidades educativas especiales."

Basándonos en la fundamentación teórica, la manera de mejorar su formación y actitudes es con un programa de "Formación+Contacto"

\subsection{Preguntas de investigación}

¿Si implementamos metodologías didácticas (aprendizaje servicio) adecuadas y facilitamos que los estudiantes participen en escenarios de aprendizaje inclusivos para el desarrollo de 
actitudes favorables a la inclusión educativa y social y para mejorar su formación inicial, constataremos cambios significativos en dichas actitudes independientemente del género y mejoras en sus resultados formativos en la asignatura de Atención a la diversidad del Grado de Educación Primaria?

\subsection{Objetivos de investigación}

\section{Objetivo General}

$\{$ Valorar la incidencia del poyecto ApS, a través de la estrategia de "Formación+Contacto" en las actitudes de los estudiantes de magisterio y en la mejora de su formación inicial en relación con la inclusión educativa y social de las personas con discapacidad.

\section{Objetivos Específicos}

$\{$ Analizar las actitudes de los estudiantes de Magisterio según su sexo hacia la inclusión social y educativa de las personas con discapacidad antes y después del proyecto.

$\{$ Analizar la formación hacia la inclusión social y educativa de las personas con discapacidad en dos grupos de estudiantes de Magisterio de ambas Facultades, una vez hayan participado uno de ellos en los diferentes escenarios inclusivos.

$\{$ Analizar, interpretar y explicar los resultados obtenidos en las pruebas pretest, postest y durante la implementación del proyecto.

\subsection{Definición de las variables de investigación}

En función del tipo de diseño, hablaremos de variables dependientes e independientes, o sólo de variables del estudio. En nuestro caso, tal y como se explica en el siguiente apartado sobre el Diseño de la Investigación, planteamos un diseño cuasiexperimental, en el que habrá manipulación de variables independientes y se medirán los efectos en las variables dependientes.

La pregunta de investigación hace referencia a la manipulación o variación de una variable, que será la principal Variable Independiente de la investigación y que tiene que ver con la participación en el proyecto ApS de la asignatura de Atención a la Diversidad de segundo curso del Grado de Maestro en Educación Primaria. Por lo tanto la variable Independiente de la investigación es la siguiente: 
1. La Variable Independiente del estudio es la "participación en el proyecto ApS".

Y la pregunta de investigación planteada anteriormente hace referencia a la medida de dos variables diferenciadas, que son las dos variables dependientes de la investigación:

2. La primera Variable Dependiente del estudio es "el nivel de incremento de la actitud de los estudiantes hacia la inclusión social de las personas con necesidades educativas especiales estrechando las diferencias existentes en función del género". 3. La segunda Variable Dependiente del estudio es "el nivel de incremento de la formación inicial de los estudiantes en los resultados de la asignatura de Atención a la Diversidad y la mejora de los mismos".

Un tema central en investigación es el control de las variables extrañas. En el apartado siguiente, al hablar del Diseño de la Investigación, se comenta la forma en que se ha procurado controlar el mayor número posible de variables extrañas que puedan influir en los valores de la Variable Dependiente.

\subsection{Diseño de investigación}

El paradigma en el que se apoya nuestra investigación, es el paradigma positivista, que ha sido el tradicionalmente dominante en la investigación en educación (Del Río, 2003) y en la educación especial (Molina, 2003).

El propósito de la investigación se encamina a la explicación, el control, la comprobación y la predicción de los fenómenos educativos. Al investigador lo que le interesa es el conocimiento observable, objetivo y cuantificable, con posibilidades de generalización.

El objetivo fundamental es explicar la relación entre variables, establecer proposiciones de causa-efecto que, de alguna forma nos permitan realizar predicciones de futuro (Del Río, 2003).

El modelo que le es propio a este paradigma es el experimental, el enfoque es el hipotético-deductivo-experimental, el método por antonomasia es de tipo cuantitativo y las técnicas que utiliza se basan en la medición y en la observación controlada, por lo que el 
campo de investigación se reduce a lo observable, a lo medible y a lo cuantificable. El método es una garantía de objetividad, y ésta junto con la validez interna y la fiabilidad conforman los criterios de calidad de la investigación desde este paradigma.

"Pero la realidad educativa, en muchos casos, impide o dificulta la asignación aleatoria de los miembros a los grupos experimental y de control; normalmente se trabaja con grupos naturales, es decir, con grupos definidos previamente, cursos, ciclo, profesores, grupos ya formados, con lo cual es muy complejo realizar cambios e igualar grupos con afán de equivalencia" (Jiménez y Tejada, 2007: 589).

Dadas las características de la investigación y la población a la que va referida, el diseño más apropiado será un diseño cuasi-experimental "pretest-postest con grupo de control no equivalente".

La investigación cuasi-experimental proviene del ámbito educacional dónde la investigación de ciertos fenómenos no podía llevarse a cabo siguiendo los procedimientos experimentales. Fueron Campbell y Stanley en 1996 quienes con la publicación del libro titulado "Experimental and Quasi-experimental Designs for Research" abordaron el estudio sistemático de los diseños cuasiexperimentales.

En los diferentes manuales consultados, existe coincidencia en señalar que las aportaciones de Campbell y Stanley y posteriormente de Cook y Campbell son las que han dado cuerpo a esta modalidad de investigación social. (García Llamas, González y Ballesteros, 2001).

\footnotetext{
“Generalmente los procedimientos de análisis de esta opción metodológica toman como referencia la investigación experimental, así podemos indicar que en este caso no se dan dos de las condiciones básicas: los sujetos no son asignados de forma aleatoria a los grupos de intervención y además los niveles de control son menores al intervenir sobre la variable independiente, puesto que depende de las peculiaridades de los sujetos antes de comenzar la investigación”. (García Llamas González y Ballesteros 2001: 290).
}

En estos diseños puede existir la manipulación de las variables independientes, pero como ya se ha señalado anteriormente, en ningún caso se da la asignación aleatoria de los sujetos a los grupos, siendo esta ausencia de aletorización su principal característica. 
(García Gállego, 2008). En nuestra investigación se ha tratado de elegir grupos que sean equivalentes, por lo menos en algunas variables (actitudes hacia la discapacidad y mismo profesor que imparte clases en todos los grupos), para que podamos ver el efecto en las variables dependientes que son las actitudes ante la inclusión social después de pasar por el programa de formación y también si ha mejorado su formación inicial. Sin embargo, no puede decirse que estos grupos sean equivalentes, de hecho pueden existir otras variables que hagan que los grupos sean distintos.

Además hemos elegido este tipo de diseño, ya que en la escuela, no se puede establecer un control riguroso, como en el verdadero experimento. Por ello al existir un menor control el investigador tiene una menor certeza sobre las inferencias de los resultados a situaciones similares. A pesar de las deficiencias en el control, los diseños cuasiexperimentales han adquirido en las últimas décadas un gran protagonismo en la investigación aplicada, fundamentalmente en el área de la investigación social, educativa y de evaluación de programas (García Gállego, 2008).

Las características de la investigación cuasi-experimental son las que más se ajustan a las condiciones en las que vamos a realizar nuestro estudio:

- Utilizamos escenarios naturales, es decir nosotros vamos a realizar nuestro estudio en situación real o de campo, la Facultad de Ciencias Humanas y de la Educación de Huesca.

- El control de los procesos es parcial, y nos permite recurrir a este tipo de investigación cuando no es posible la utilización de la metodología experimental en sentido estricto, ya que hay un control parcial de ciertas variables extrañas.

- La manipulación del investigador sobre la variable independiente, en las condiciones en que lo permita la situación, por lo que es posible que algunas variables hayan quedado sin controlar debidamente.

- Tiene grandes posibilidades de aplicación al campo social y educativo, cuando no se puede llegar a un nivel más riguroso.

A pesar de estas limitaciones, estos diseños están ampliamente difundidos en la investigación educativa. Campbell y Stanley presentan seis tipos de diseños cuasiexperimentales diferentes, uno de los más interesantes es el que se utiliza en esta investigación, que es un diseño pretest postest con grupo control no equivalente, pero 
contrabalanceado en la variable actitud hacia la inclusión escolar. Diseño que Jiménez y Tejada (2007) consideran muy interesante para la investigación en la escuela.

\section{Desarrollo del Proyecto}

En el curso escolar 2012-2013 en el Grado de Magisterio de la Universidad de Zaragoza y en concreto en la Facultad de Educación de Zaragoza y en la Facultad de Ciencias Humanas y de la Educación de Huesca llevamos a cabo una investigación cuyo objetivo fue analizar las actitudes de los estudiantes de segundo curso del Grado de Maestro en Educación Primaria hacia la inclusión de las personas con necesidades educativas especiales.

Se utilizó la escala de actitudes de Verdugo, Arias y Jenaro (1994), en una muestra de 180 alumnos todos ellos de segundo curso. Parte de ellos fueron el grupo control y el resto el grupo experimental. El grupo experimental se constituyó con los alumnos de la Facultad de Huesca: de los alumnos del espacio muestral, aquellos que participan en el proyecto de Aprendizaje Servicio.

El grupo control: los alumnos de la Facultad de Zaragoza que no participaron en dicho proyecto.

La investigación tiene un carácter cuasi-experiental, basada en un pretest y en un postest. Se comparará el pretest y postest intragrupos (los que han participado en el proyecto vs los que no han participado).

En febrero del curso 2012-2013, realizamos una valoración de la similitud de los grupos en el pretest, para garantizar la homogeneidad entre los grupos antes de comenzar el proyecto. En esa primera valoración los estudiantes tenían actitudes similares hacia la inclusión escolar de las personas con necesidades educativas especiales sin manifestar diferencias significativas entre los grupos, nos llamó la atención que la actitud de los alumnos varones era significativamente menos positiva que la actitud de las mujeres, mostrando actitudes mucho menos integradoras y basadas en teorías deficitarias.

Apoyándonos en la revisión de las principales referencias científicas sobre la potenciación de actitudes positivas hacia la inclusión, Skrtic, Clark y White (1982), Verdugo y Arias (1991), Slininger, Sherrill y Jankowski (2000), Duquette (2000), Folson-Meek, Nearing y Kalakian (2000), Hodge, Davis, Woodard y Sherrill (2002), McCarth y Misquez (2003), 
Aguado, Flórez, Alcedo (2003, 2004) y Aguado, Alcedo, Arias (2008), hemos diseñado el proyecto de ApS de intervención educativa en la asignatura de Atención a la Diversidad, usando la estrategia que mejores resultados ha dado, que es la "Formación+Contacto" de los estudiantes con las personas con necesidades educativas especiales. La "formación” se realizó con las sesenta horas de la asignatura de Atención a la Diversidad, y el "contacto" se basó en veinte horas de prácticas en los diferentes escenarios de las distintas asociaciones de discapacidad de Huesca y provincia. La duración fue de seis meses, haciendo simultáneamente la formación teórica en la asignatura y dos horas a la semana de prácticas en una asociación de atención a las personas con necesidades educativas especiales.

El grupo experimental estuvo constituido por noventa y ocho alumnos y el grupo control los ochenta y dos restantes pertenecientes a la Facultad de Educación de Zaragoza que no han participado en el proyecto ApS.

La intención de nuestra investigación fue verificar si es posible el establecimiento de una relación entre la aplicación de un programa de intervención educativa basado en la estrategia de "formación+contacto" con una duración de ochenta horas (variable dependiente), y el incremento o descenso de las actitudes favorables a la inclusión de las personas con necesidades educativas especiales en la sociedad y en la escuela independientemente del género. Además de verificar si hay un aumento o disminución del rendimiento de los estudiantes en la asignatura de Atención a la Diversidad del Grado de Primaria.

Para evaluar las actitudes ante las necesidades educativas especiales se utilizó la "Escala de Actitudes hacia las Personas con Discapacidad. Forma G” (Verdugo, Arias y Jenaro, 1994). Se trata de una escala de evaluación de actitudes ante personas con cualquier tipo de discapacidad formada por treinta y siete ítems.

La escala fue aplicada a los estudiantes durante una de las primeras clases del segundo cuatrimestre de 2013. Se dieron las instrucciones estandarizadas que aparecen en el protocolo de la misma y se aclararon las dudas que algunos sujetos manifestaron. Dispusieron del tiempo que necesitaron para la total cumplimentación de la escala. Las respuestas se analizaron con el paquete estadístico SPSS 12.0 y manifestaron que no había diferencias significativas entre el grupo control y el experimental antes de iniciar el programa en la actitud que tenían los estudiantes hacia las personas con discapacidad, salvo en la variable género dónde se apreciaban diferencias significativas entre los estudiantes con género masculino y femenino, 
teniendo este último grupo actitudes más inclusivas hacia la diversidad. Este análisis ya había sido puesto sobre la mesa por otras investigaciones.

\section{Evaluación}

Consideramos que deben evaluarse como índices de calidad de la investigación los dos apartados siguientes:

1. Evaluación de las fases de la investigación.

2. Evaluación de los resultados de la implementación de metodología didáctica y de la participación en el proyecto de ApS.

Estos dos primeros puntos tienen que ver con el efecto de tratamiento (efecto producido por la variable independiente en las variables dependientes), por la Sensibilidad de la Investigación (probabilidad de que un efecto, si está realmente presente, pueda ser detectado) y por la Validez de la Investigación (determina la calidad del diseño de la investigación, haciendo referencia a la veracidad de los datos obtenidos en relación a las variables que se querían medir). Deben analizarse las amenazas contra la validez, diferenciando entre validez interna de constructo y externa.

Esta evaluación nos facilitará indicadores para valorar la incidencia y necesidad de ofertar estos complementos formativos (teórico-prácticos y de convivencia) en los estudios de grados de maestro. Igualmente arrojará datos que nos permitirán comprobar si estos complementos, tal como los tenemos diseñados son suficientes o si es necesario incluir alguna mejora o complemento más.

\section{Discusión de Resultados y Conclusiones}

Tras pasar la "Escala de Actitudes hacia las Personas con Discapacidad" en mayo de 2013 a los estudiantes de Magisterio que participaron en el proyecto ApS, hemos obtenido los siguientes resultados: 
En primer lugar, se han encontrado diferencias significativas entre el grupo control y el experimental en especial en algunas subescalas del instrumento, que pasamos a comentar.

Los estudiantes del grupo experimental en el postest manifestaron una actitud positiva ante las necesidades educativas especiales sin diferencias significativas en la variable sexo, que si habían sido detectadas en el pretest. Esto nos lleva a pensar que el proyecto de ApS ha sido exitoso y ha conseguido uno de los objetivos fundamentales que pretendía, eliminar esa diferencia.

El 78\% de los estudiantes del grupo experimental obtuvieron puntuaciones más bajas en la subescala relacionada con "las limitaciones de las personas con necesidades educativas especiales", dónde se afirma que "son menos inteligentes que las personas sin necesidades educativas especiales, que sólo pueden hacer trabajos sencillos y repetitivos, que sólo pueden seguir instrucciones simples, que funcionan como los niños y que de ellos no se puede esperar demasiado y además son poco constantes". Poniendo de manifiesto que la actitud del profesorado cuando es negativa es una de las principales barreras a la integración de las personas con necesidades educativas especiales en la escuela y en la sociedad.

En lo que se refiere a la valoración de las capacidades de las personas con necesidades educativas especiales, el grupo experimental en un 89\% respondió afirmativamente al ítem que dice "que las personas con necesidades educativas especiales son competentes, constantes y que no tienen problemas con sus compañeros", en cambio en el grupo control tan sólo afirmaron este ítem el 53\%. Por lo que las actitudes positivas hacia las personas con necesidades educativas especiales están muy relacionadas con las expectativas que el maestro tiene hacia ellas, y esto lo podemos relacionar con el trabajo experimental de Rosenthal y Jacobson, Pygmalión en la escuela (1968), dónde se hace referencia al mito griego del escultor que se enamora de una de sus obras, tratándola como si estuviera viva de tal forma que termina estándolo, haciendo así realidad una creencia. La máxima de este estudio es "quien cree en algo lo consigue" o "quien cree que alguien puede hacer algo al final lo hace", en cambio "el maestro que no cree en las capacidades de sus alumnos impide que estos progresen al ritmo que podrían hacerlo". Por ello las actitudes positivas de los maestros hacia los alumnos con necesidades educativas especiales son tan importantes, pues podrían condicionar la integración de estos en las aulas y posteriormente en la sociedad. 
Y por eso desde los años 70 se está investigando en los países anglosajones influenciados por el Informe Warnock y el concepto de normalización, el cambio de actitudes hacia la diversidad.

En este estudio, se ha encontrado también diferencias significativas entre el grupo control y el experimental en los ítems dónde se reconocen los derechos de las personas con necesidades educativas especiales al empleo, al ocio, a tener una pareja, a tener una vivienda propia, a favor de los alumnos que han participado en el proyecto. Las personas del grupo de control tienen una visión más estereotipada de las necesidades educativas especiales en este sentido.

En los ítems dónde se niegan algunos derechos fundamentales a las personas con necesidades educativas especiales, no se encuentran diferencias significativas entre los estudiantes que han participado en el proyecto y los que no han participado en el mismo.

En la subescala de "Asunción de Roles" dónde se valora la autoestima que tiene la persona con necesidades educativas especiales, si tiene confianza en sí misma, en su valor, y si se siente capaz de llevar una vida normal. En estos ítems las diferencias entre el grupo control y el experimental son significativas a favor del grupo experimental. Pues en el grupo control un $87 \%$ percibe que las personas con discapacidad tienen bajos niveles de autoestima, mientras que en el grupo experimental más de un 50\% consideran que la mayoría de las personas con necesidades educativas especiales tienen confianza en sí mismas y con los apoyos necesarios se sienten capaces de llevar una vida lo más normalizada posible, aumentando así su autoestima. En este sentido, recordar lo importante que son las actitudes del maestro, Florez et. al, (2009) incidían en ello al afirmar que las actitudes que tiene el maestro hacia la persona con necesidades educativas especiales influyen en su autoestima y en sus posibilidades personales.

Otro resultado a destacar, es que es más positiva la actitud ante las necesidades educativas especiales de los estudiantes que han realizado sus prácticas en asociaciones que trabajan con discapacidad sensorial (auditiva o visual) y motórica, que aquellos que han realizado sus prácticas en asociaciones vinculadas a la discapacidad intelectual o a la enfermedad mental. Estos datos concuerdan con los encontrados por Moreno, Rodríguez, Saldaña y Aguilera (2006), donde las actitudes más negativas están asociadas a retraso mental. En las conversaciones con nuestros estudiantes también se ha refutado este resultado, los alumnos manifiestan actitudes más negativas hacia la discapacidad cuando han mantenido 
contacto con personas con enfermedad mental, que los que han mantenido contacto con otras discapacidades.

Por otro lado, también hemos constatado mejora en los resultados en la asignatura de atención a la diversidad de los alumnos que han participado en el proyecto de ApS, comparándolo con el grupo de control. En el grupo experimental un $87 \%$ aprobaron la asignatura mientras que el grupo de control este porcentaje era del $76 \%$. Teniendo el grupo experimental calificaciones mucho más altas.

Una vez presentado los principales resultados y teniéndolos en consideración, debemos descartar que se confirma nuestra hipótesis de partida, que a mayor formación la actitud de los estudiantes independiente de su género es más positiva hacia la inclusión educativa y social de las personas con necesidades educativas especiales, además de que las diferencias actitudinales entre los varones y mujeres disminuyen. Y así mismo, se confirma que mejoran los resultados de los estudiantes en la asignatura de Atención a la Diversidad del Grado de Educación Primaria.

Nuestro estudio se ha apoyado en investigaciones como la realizada por Muratori, Guntín y Delfino (2010) donde se concluyó que el contacto con personas con discapacidad ejerce influencia sobre las actitudes hacia éstas personas, los estudiantes que tienen esta experiencia, manifiestan actitudes más positivas que los que reconocen no tener contacto. Así mismo, detectaron que a mayor frecuencia del contacto, la valoración era más positiva acerca de los derechos, capacidades y limitaciones de las personas con discapacidad. Recientemente Suriá (2011) afirmó que los estudiantes están más sensibilizados hacia las necesidades educativas especiales cuando interactúan con compañeros con discapacidad. Teniendo en cuenta lo anterior y con los resultados encontrados en el postest de este estudio, apostamos por facilitar espacios de encuentro y convivencia entre nuestros alumnos, propiciando el contacto con discapacidades de toda índole, que potencien un cambio de actitudes y por lo tanto supongan una mejora de nuestra sociedad. Del mismo modo, pone de manifiesto que la actitud positiva ante la diversidad se potencia si el contacto se establece con personas con discapacidad sensorial y motórica, que no esté asociada a discapacidad intelectual, ni a enfermedad mental.

Sucintamente, con esta investigación hemos querido potenciar que los futuros docentes independientemente de su sexo sean competentes y desarrollen estrategias para trabajar con la diversidad del aula y del centro educativo, además de limar las diferencias actitudinales que hemos encontrado entre varones y mujeres, y que algunas investigaciones a las que hemos hecho referencia ya las habían detectado. A lo largo de este artículo hemos puesto de manifiesto que la 
formación inicial del maestro/a generalista influye en la actitud hacia la inclusión escolar y social de las personas con necesidades educativas especiales. El maestro/a tutor de un aula ordinaria es pieza clave en la educación de todos sus alumnos, y entendemos que la formación en atención a la diversidad que reciben los estudiantes de Magisterio es muy escasa, por eso hemos querido ampliar dicha formación, fundamentándonos y apoyándonos para ello en investigaciones como las realizadas por Wehmeyer, Agran y Hughes (2000), Gughwan y Chow (2001), Arranz (2002, 2003), Yazbeck, McVilly y Parmerter (2004), Arranz, Herrero y Liesa (2006), Liesa y Vived (2009), Oullette-Kuntz, Burge, Brown y Arsenault (2010), y Vigo, Soriano y Julve (2010) que han demostrado que un mayor nivel de estudios y formación permite tener una visión y un conocimiento más real sobre las necesidades educativas especiales, y por lo tanto actitudes más favorables tanto en los maestros varones como en las maestras mujeres.

En este sentido, la formación inicial y continua del maestro generalista es muy importante para que su actitud hacia la inclusión escolar y social de las personas con necesidad de apoyo educativo sea positiva, ya que el maestro tutor de un aula ordinaria es pieza clave en la educación de todos sus alumnos. Por este motivo, entendemos que la formación en atención a la diversidad que reciben en el Grado es muy escasa y diseñamos proyectos con las asociaciones del entorno próximo dónde puedan ampliar dicha formación.

Así mismo y de acuerdo con los resultados encontrados en este trabajo, pretendemos concienciar a los órganos competentes en los diseños curriculares de la titulación de Magisterio de esta necesidad formativa, para que introduzcan las actitudes del profesorado como competencias a trabajar en los currículums y proyectos docentes. Son escasas las instituciones y centros educativos que incluyen entre sus finalidades competenciales el enseñar a los futuros maestros contenidos dirigidos a mejorar las actitudes ante la diversidad y la capacidad de evaluar dichas actitudes, a pesar de que estas competencias y actitudes están contempladas en la formación y el ejercicio de la profesión del educador (ANECA, 2004).

Como conclusión general, para favorecer que se produzca este cambio actitudinal hacia la discapacidad, en nuestra Facultad apostamos por la utilización de la estrategia ("formación+contacto") con el colectivo de futuros maestros. Nos hemos apoyado en una metodología constructivista y experiencial, porque pretendemos que el alumnado realice aprendizajes significativos, ya que son estos, los que se relacionan directamente con el proyecto que hemos puesto en marcha a través de la metodología de ApS. 


\section{BIBLIOGRAFÍA}

- Aguado, Antonio-León; Alcedo, Ma Ángeles y Arias, Benito (2008): “Cambio de actitudes hacia la discapacidad con escolares de Primaria”. En: Psicothema, n² 20, 4, pp. 697-704.

- Aguado, Antonio-León; Flórez García, María Ángeles y Alcedo, Ma Ángeles (2003): “Un programa de cambio de actitudes hacia personas con discapacidad en entorno escolar". En: Análisis y Modificación de Conducta, no 29 (127), pp. 673-704.

. (2004): "Programas de cambio de actitudes ante la discapacidad". En: Psicothema, $\mathrm{n}^{\mathrm{o}} 16$ (4), pp. 667-673.

- Aneca (2004): Libro Blanco del Título de Grado en Magisterio. Agencia Nacional de Evaluación de la Calidad y Acreditación.

- Arranz, Pilar (2002): "El pensamiento de los maestros a cerca de la integración de los alumnos con Síndrome de Down en el aula ordinaria. Elementos favorecedores del éxito de la misma”. En: Anuario de Pedagogía, no 4, pp. 47-78.

(2003): "La formación inicial del profesorado: modelos, demandas y prescripciones". En: Anuario de Pedagogía, nº 5, pp. 75-101.

- Arranz, Pilar; Herrero, Marisa y Liesa, Marta (2006): "Necesidad de formación del profesorado en la atención a la diversidad: un proceso de investigación-acción”. En Cifuentes, M.A. y otros (Coords.). La accesibilidad como medio para educar en la diversidad: educación, diversidad y accesibilidad en el entorno europeo. Burgos: Octaedro. pp. 429-440.

- Astin, Alexander W. et al. (2000). How Service Learning Affects Students. UCLA: Higher Education Research Institute.

- Avramidis, Elias y Norwichi, Brahm (2004): "Las actitudes de los profesores hacia la integración y la inclusión: revisión de la bibliografía sobre la materia”. En: Entre dos mundos: revista de traducción sobre discapacidad visual, $\mathrm{n}^{\circ} 25$, agosto, pp. 25-44.

- Campbell, Donal .J. y Stanley, Julian. C. (1996): Experimental and Quasi-experimental designs for research. Chicago: Rand McNally College Publishing Company.

- Del Río, Dionisio (2003): Métodos de investigación en educación. Procesos y diseños no complejos. Vol. I. Madrid: Uned.

- Dengra, Ramón; Durán, Remedios y Verdugo, Miguel Ángel (1991): "Estudio de las variables que afectan a las actitudes de los maestros hacia la integración escolar de niños con 
necesidades especiales". En CEPE: Psicopedagogía terapéutica. Anuario español e iberoamericano de investigación en educación especial, pp. 47-88.

- Downs, Peter y Wiliams, Trevor (1994): "Students attitudes toward integration of people with disabilities activity settings". En: Adapted Physical Activity Quartely, no 11(1), pp. 32-43.

- Evans, Joseph H. (1976): “Changing attitudes toward disbled persons: An experimental study". En: Rehabilitation Counseling Bulletin, n 19, pp. 572-579.

- Ferrán, Anne y Guinot, Cintia (2012): “Aprendizaje-servicio: propuesta metodológica para trabajar competencias”. En Portularia, vol. XII, nº extra, pp. 460-479.

- Flórez, María Ángeles; León Aguado, Antonio y Alcedo, María Ángeles (2009): "Revisión y análisis de los programas de cambio de actitudes hacia personas con discapacidad". En Anuario de Psicología Clínica y de la Salud, nº 5, pp. 85-98.

- Folsom-Meek, Sherry L. y Rizzo, Terry L. (2002): "Validating the physical educators' attitude toward teaching individuals with disabilities - III (PEATID-III) for future teachers". En Adapted Physical Activity Quarterly, no 19, pp. 141-154.

- Folsom-Meek, Sherry L; Nearing, Ruth y Kalakian, Leonard H. (2000): "Effects of an adapted physical education course in changing attitudes". En: Clinical Kinesiology, no 54(3), pp.52-58.

- Furco, Andrevv (2004): "El impacto educativo del aprendizaje-servicio. Ponencia en el VII Seminario Internacional de aprendizaje y servicio solidario". Buenos Aires, 6-7 octubre de 2004. Ministerio de Educación, Ciencia y Tecnología, República Argentina. En prensa.

- García Gállego, Carmen (2008): “Investigación cuasiexperimental I: Diseños preexperimentales y diseños cuasiexperimentales con grupo de control no equivalente". En Fontes de Gracia, S. et. al. Diseños de investigación en psicología. Madrid: Uned.

- García Llamas, José Luis; González Galán, María Ángeles y Ballesteros Velázquez, Belén (2001): Introducción a la investigación en educación. Tomo II. Madrid: Uned.

- Gughwan, Choi y Chow, Lam (2001): “Korean students' differential attitudes toward people with disabilities: an acculturation perspective". En: International Journal of Rehabilitation Research, $\mathrm{n}^{\circ}$ 24, pp. 79-81.

- Hodge, Samuel R.; Davis, Ronald; Woodard, Rebecca y Sherrill, Carol (2002): "Comparison of practicum types in changing preservice teacher's attitudes and perceived competence”. En: Adapted Physical Activity Quarterly, nº 19, pp. 155-171. 
- Infante, Marta y Gómez, Viviana (2004): “Actitudes de los estudiantes de educación hacia la integración de personas con discapacidad y hacia la educación multicultural”. En: $C$ y $E$ : Cultura y Educación. Vol. 16, nº 4, pp. 371-384.

- Jiménez, Blanca y Tejada, José (2007): “Procesos y métodos de investigación”. En Tejada, J. (Coord.). Formación de Formadores. Madrid: Thomson Editores. pp. 543-625.

- Lazar, Alfred L.; Orpet, Russell y Demos, George (1976): "The impact of class instruction on changing student attitudes”. En: Rehabilitation Counseling Bulletin, nº 20, pp. 66-68.

- Liesa, Marta y Vived, Elias (2009): "Desarrollo de las competencias de los maestros en atención a la diversidad a través de diseño de escenarios de inclusión social". En: Revista Argentina de Psicopedagogía, nº 62, pp 30-34.

- Molina, Santiago (2003): Educación Especial. Bases Metateóricas e Investigadoras. Granada: Arial Ediciones.

- Moreno, Francisco Javier; Rodríguez, Isabel; Saldaña, David y Aguilera, Antonio (2006): "Actitudes ante la discapacidad en el alumnado universitario matriculado en materias afines". En: Revista Iberoamericana de Educación, no 40 (5-25), pp. 1-12.

- Muratori, Marcela; Guntín, Carolina y Delfino, Gisela (2010): “Actitudes de los adolescentes hacia las personas con discapacidad: un estudio con alumnos del polimodal en la zona norte del conurbano bonaerense" [en línea]. En: Revista de Psicología, nº 6 (12), pp. 3956, Disponible en: http://bibliotecadigital.uca.edu.ar/repositorio/revistas/actitudesadolescentes-hacia-personas-discapacidad.pdf [25/01/2014].

- Novo Corti; Isabel y Muñoz Cantero, Jesús Miguel (2012): “Los estudiantes universitarios ante la inclusión de sus compañeros con discapacidad: indicadores basados en la teoría de la acción razonada para los estudios de economía y empresa en la universidad de A Coruña (España)" En: REOP. Vol. 23, no 2, $2^{\circ}$ Cuatrimestre, 2012, pp.105-122.

- Oullette-Kuntz, Hélène; Burge, Philip; Brown, Hilary K. y Arsenault, Elizabeth (2010): "Public attitudes towards individuals with intellectual disabilities as measured by the concept of social distance”. En: Journal of Applied Research in Intellectual Disabilities, $\mathrm{n}^{\mathrm{o}}$ 23(2), pp.132-142.

- Pelechano, Vicente (dir.) (1990): Aceptación, habilidades sociales y motivación en la integración de niños ciegos. Informe técnico. Tenerife: Departamento de Personalidad, Universidad de La Laguna. 
- Pelechano, Vicente; García, Liria y Hernández, Ascensión (1994): “Actitudes hacia la integración de invidentes y habilidades interpersonales: Planteamiento y resultados de dos programas de modificación". En: Integración, n 15, pp. 5-22.

- Pelechano, Vicente; Peñate, Wenceslao y De Miguel, Adelia (1991): “Actitudes hacia la integración de invidentes y personalidad”. En: Análisis y modificación de conducta, n 17 (5354), pp. 439-456.

- Puig Rovira, Josep $\mathrm{M}^{\mathrm{a}}$ et al. (2011): “Aprendizaje-servicio y Educación para la Ciudadanía”. En: Revista de Educación, pp. 45-67.

- Puig, Josep M $\mathrm{M}^{\mathrm{a}}$ y Palos, Josep (2006): “Rasgos pedagógicos del aprendizaje-servicio”. En: Cuadernos de Pedagogía, no 357, pp. 60-63.

- Puig, Josep Má Batlle, Roser; Bosch, Carme y Palos, Josep (2007): Aprendizaje Servicio: educar para la ciudadanía. Barcelona, Octaedro, [en línea] (primer capítulo) Disponible en http://www.zerbikas.es [25/11/2013].

- Rial, Sergio (2010): “Criterios de calidad y rasgos característicos de las experiencias de aprendizaje-servicio en la educación formal”. Tzhoecoen. Perú: Universidad Señor de Sipan de Chiclayo.

- Sáenz, Oscar (1990): “Actitudes de los profesores ante la integración del niño discapacitado en la escuela ordinaria". En: Revista Interuniversitaria de Formación del Profesorado, n 8, pp. 135-150.

- Sales, Auxiliadora; Moliner, Odet. y Sanchiz, María Luisa (2001): “Actitudes hacia la atención a la diversidad en la formación inicial del profesorado". En: Revista electrónica Interuniversitaria de Formación del Profesorado, $\mathrm{n}^{\circ} 4$ (2), pp. 1-7.

- Slininger, Drew; Sherrill, Carol y Jankowski, Catherine (2000): Children's attitudes toward peers with severe disabilities: Revising contact theory. En: Adapted Physical Activity Quarterly, n 17, pp. 176-196.

- Stanton, Timothy (1990): "Service Learning: Groping toward a definition" in Kendall et al., Combinig Service and Learning: A resource Book for Community and Public Service (Vol. 1), pp. 65-67.

- Stephenson, Meg; Wechsler, Ann y Welch, Marshall (2002): Service Learning in the Curriculum: a faculty guide. Utah: Lowell Bennion Community Service Center at the University of Utah. 
- Surriá Martínez, Raquel (2011): “Análisis comparativo de las actitudes de los estudiantes hacia sus compañeros con discapacidad”. En: Electronic Journal of Research in Educational Psychology, no 9 (1), 23, pp.197-216.

- Tapia, María Nieves (2000): La Solidaridad como Pedagogía. Buenos Aires: Ciudad Nueva. (2008): "Calidad académica y responsabilidad social: el aprendizaje servicio como puente entre dos culturas universitarias" en Martínez, M. (coord). Aprendizaje servicio y responsabilidad social de las Universidades. Barcelona: Octaedro. pp. 27-56.

- Verdugo, Miguel Ángel y Arias, Benito (1991): “Evaluación y modificación de las actitudes hacia los minusválidos". En: Revista de Psicología General y Aplicada, n 44, (1), pp. 95-102.

- Verdugo, Miguel Ángel; Arias, Benito y Jenaro, Cristina (1994): Actitudes hacia las personas con minusvalía. Madrid: Ministerio de Asuntos Sociales, Instituto Nacional de Servicios Sociales.

. (1995): “Actitudes sociales y profesionales hacia las personas con discapacidad: estrategias de evaluación e intervención”. En M. A. Verdugo y Aguado. Personas con discapacidad: perspectivas psicopedagógicas y rehabilitadoras. México: Siglo XXI. pp. 79143.

- Vigo, María Begoña; Soriano, Juna y Julve, María Carmen (2010): "Preparando profesionales para la atención a la diversidad: potencialidades y limitaciones de un proyecto de innovación y mejora interdisciplinar". En: Revista interuniversitaria de formación del profesorado, $\mathrm{n}^{\circ} 67$, pp. 147-166.

- Warnock, Mary (1978): "Special Educational Needs. Report of the committee of enquiry into the education of handicapped children and young people”. London: HMSO. Traducido al español. En: Siglo Cero, n ${ }^{\circ}$ 130, pp. 12-24. Informe sobre necesidades educativas especiales.

- Wehmeyer, Michael L.; Agran, Martin y Hughes, Carolyn (2000): "A national survey of teachers'promotion of self-determination and student directed learning". En: The Journal of Special Education, $\mathrm{n}^{\mathrm{o}} 34$ (2), pp. 58-68.

- Yazbeck, Marie; McVilly, Keith y Parmenter, Trevor (2004): “Attitudes toward people with intellectual disabilities”. En: Journal of Disability Policy Studies, n 15 (2), pp. 97-112. 